\title{
Carbon burning in intermediate-mass primordial stars
}

\author{
P. Gil-Pons ${ }^{1}$, T. Suda ${ }^{2,3}$, M. Y. Fujimoto ${ }^{2}$, and E. García-Berro ${ }^{1,4}$ \\ ${ }^{1}$ Departament de Física Aplicada, Escola Politécnica Superior de Castelldefels, Universitat Politècnica de Catalunya, \\ Avda. del Canal Olímpic s/n, 08860 Castelldefels, Spain \\ e-mail: [pilar;garcia]@fa.upc.es \\ ${ }^{2}$ Department of Physics, Hokkaido University, Sapporo, Hokkaido 060-0810, Japan \\ 3 Meme Media Laboratory, Hokkaido University, Sapporo, Hokkaido 060-0813, Japan \\ ${ }^{4}$ Institute for Space Studies of Catalonia, c/Gran Capità 2-4, Edif. Nexus 104, 08034 Barcelona, Spain
}

Received 29 July 2004 / Accepted 2 December 2004

\begin{abstract}
The evolution of a zero metallicity $9 M_{\odot}$ star is computed, analyzed and compared with that of a solar metallicity star of identical ZAMS mass. Our computations range from the main sequence until the formation of a massive oxygen-neon white dwarf. Special attention has been payed to carbon burning in conditions of partial degeneracy as well as to the subsequent thermally pulsing Super-AGB phase. The latter develops in a fashion very similar to that of a solar metallicity $9 M_{\odot}$ star, as a consequence of the significant enrichment in metals of the stellar envelope that ensues due to the so-called third dredge-up episode. The abundances in mass of the main isotopes in the final ONe core resulting from the evolution are $X\left({ }^{16} \mathrm{O}\right) \approx 0.59$, $X\left({ }^{20} \mathrm{Ne}\right) \approx 0.28$ and $X\left({ }^{24} \mathrm{Mg}\right) \approx 0.05$. This core is surrounded by a $0.05 M_{\odot}$ buffer mainly composed of carbon and oxygen, and on top of it a He envelope of mass $\sim 10^{-4} M_{\odot}$.
\end{abstract}

Key words. stars: evolution - stars: abundances - stars: AGB and post-AGB - stars: interiors

\section{Introduction}

The primordial universe was mainly composed of hydrogen $(X \approx 0.77)$, helium $(Y \approx 0.23)$, and some ${ }^{2} \mathrm{H},{ }^{3} \mathrm{He}$ and ${ }^{7} \mathrm{Li}$ in very small amounts - the total abundance did not represent more than $10^{-10}$ of the total mass of the universe. This set of abundances, derived from the standard homogeneous Big Bang nucleosynthesis model (Alpher \& Herman 1950; Olive 1999), characterizes the matter out of which the first structures of the Universe formed. According to the accepted hierarchical scenario, these large structures had a mass between $10^{5}$ and $10^{8} M_{\odot}$, and very soon after their formation became the nest in which primordial stars formed.

There is no general agreement on the initial mass function (IMF) of these primordial stars. Instead, this particular subject is still today a matter of strong debate. Moreover, the original composition of these stars was definitely determined by the fragmentation and condensation processes that led to their formation and, therefore, it is closely related to the adopted IMF. The first attempts in this direction pointed to the dominance of very massive stars. The reason was the original lack of metallic molecules that allowed the cooling necessary to form low and intermediate mass objects. Therefore, much of the earliest theoretical work on $Z=0$ stars was devoted to the study of very massive objects - see, for instance, Ezer \& Cameron (1971) and Carr et al. (1983). However, as it was claimed later, the presence of molecular hydrogen could allow the cooling necessary for low and intermediate mass objects to be formed (Carlberg 1981; Palla et al. 1983; Yoshii \& Saio 1986). This result motivated the work of D'Antona (1982), Guenter \& Demarque (1983) and Fujimoto et al. (1984) on intermediate mass primordial stars. The latest two-dimensional simulations (Nakamura \& Umemura 2001) have found that a bimodal IMF peaking at $\approx 2 M_{\odot}$ and at a mass between 10 and $100 M_{\odot}$ could be appropriate. Consequently, the most recent publications study both massive (Heger et al. 2001; Heger \& Woosley 2000) and lowintermediate mass objects (Marigo et al. 2001; Chieffi et al. 2001). For instance, Heger et al. (2000) considered the evolution of $Z=0$ stars of masses between 15 and $250 M_{\odot}$, from their main sequence until core collapse. They concluded that massive objects in general were important in the production of primordial ${ }^{14} \mathrm{~N}$ and that those stars within the highest mass range were the place were primordial $\mathrm{Fe}$ and $\mathrm{Ni}$ could be synthetized - see also Goriely \& Siess (2002) and Abia et al. (2001). Using the results of Limongi et al. (2000) and Chieffi et al. (2001) for Population III stars, Abia et al. (2001) analyzed the impact of primordial stars on polluting the intergalactic medium (IGM), and showed the relevance of an IMF peaking in the range of masses between 4 and $8 M_{\odot}$ to explain the large $[\mathrm{C} / \mathrm{Fe}]$ and $[\mathrm{N} / \mathrm{Fe}]$ ratios observed in many extremely metalpoor stars.

In a pioneering work on low and intermediate-mass stars of very low metallicity, Fujimoto et al. (1984) studied by means of semianalytical techniques the generation of helium-shell 
flashes during the AGB stage, as a function of mass and metallicity. They found that stars whose ZAMS mass was lower than $4 M_{\odot}$ experienced helium shell flashes and were important contributors of ${ }^{12} \mathrm{C}$ and neutron-rich isotopes of light elements, whereas stars with larger masses were more likely to become supernovae and, hence, to contribute substantially to the production of Fe and Ni. Marigo et al. (2001) and Marigo (2002) also studied $Z=0$ stars in the mass range $0.7 \lessgtr M / M_{\odot} \lessgtr 100$, and followed their evolution from the main sequence until the AGB phase for objects in the low- and intermediate-mass range. In particular, they considered the pollution of stellar atmospheres and, ultimatelly, of the interstellar medium, as a consequence of the dredge-up processes ensuing during the AGB phase. For the high-mass range, namely stars with masses $M_{\text {ZAMS }} \gtrsim 9 M_{\odot}$, Marigo et al. (2002) followed the evolution of their model stars until the carbon burning phase. It is, however, important to stress at this point that in the high-mass range carbon is burnt in non-degenerate conditions. Chieffi et al. (2001) focused on $Z=0,4 \lesssim M / M_{\odot} \lesssim 8$ stars and also followed the thermally pulsing phase and the dredge-up during the AGB phase. These authors concluded that stars in this mass range were the main contributors of nitrogen and oxygen to the IGM. Finally, Siess et al. (2002) also followed the evolution and nucleosynthesis of primordial stars of masses ranging between 0.8 and $20 M_{\odot}$. Their results, in agreement with those of Chieffi et al. (2001), pointed to a standard AGB behaviour for stars with masses larger than about $5 M_{\odot}$. These authors have also discussed the effects of taking into account the effects of a moderate amount of overshooting.

One might argue the scarcity of observational support for the study of $Z=0$ objects. In fact, the detection of primordial composition objects should take us back to $[\mathrm{Fe} / \mathrm{H}] \approx$ -8.3 epochs, whereas, up to now, the oldest stars detected are a red giant at $[\mathrm{Fe} / \mathrm{H}] \approx-4.6$ (Bessel \& Norris 1984), and a low mass star, HE0107-5240, with $[\mathrm{Fe} / \mathrm{H}] \approx-5.3$ (Christlieb et al. 2002). Nevertheless, as correctly pointed out by Chieffi et al. (2001), the lack of observations should not let us discard the existence of $Z=0$ intermediate mass stars. Therefore, in this paper we study the evolution of intermediate mass primordial stars.

In order to remain consistent with our previous results, we have used for the calculations reported in this paper the same evolutionary code as that described in Ritossa et al. (1995). The interested reader can find there a thorough description of the most relevant physical inputs (neutrino energy loss rates, reaction rates ...). In particular, the treatment of convective boundaries turns out to be important for the calculations reported here. We have not adopted any overshoot and convective regions were computed according to the standard procedure given by the mixing length theory in order to adequately compare our results with the calculations reported in Ritossa et al. (1995). The only major difference between the physics used there and those used in the present calculations is that, in order to properly compute the evolution of $Z=0$ model star sequences, we have implemented the corresponding opacities at very low metallicities of Iglesias \& Rogers (1993).

The plan of this paper is the following: in Sect. 2, we study the evolution of a zero metallicity $9 M_{\odot}$ model star until the beginning of carbon burning, and we compare it with the evolution of the corresponding solar metallicity model star and with the results of previous calculations. In Sect. 3, the carbon burning phase, the associated thermonuclear flashes, the dredge-up of nuclear processed elements and the associated changes in the surface composition of the star are studied. Section 4 is devoted to the study of the thermally-pulsing Super-AGB (TP-SAGB) phase. In Sect. 5, the evolution through the post-AGB track and the final phases of the evolution to become a white dwarf are described. Finally, Sect. 6 is devoted to summarizing our main results and to outline the conclusions derived from them.

\section{Hydrogen and helium burning}

We consider a zero metallicity $9 M_{\odot}$ star. As we will see below, the characteristics of the evolution during the main hydrogen and helium burning phases show important differences with respect to the evolution of analogous cases of higher metallicity. In particular, as already noted by other authors that have studied the main sequence phase of intermediate mass primordial stars - namely Chieffi \& Tornambé (1984), Tornambé \& Chieffi (1986) and Cassisi \& Castellani (1993) - the first ascent to the red giant branch does not take place before central helium ignition but, instead, it occurs when helium burning is established in a shell.

The main characteristic of $Z=0$ stars, that is, the absence of metals and, in particular, of carbon, nitrogen and oxygen, determines the evolution from its very early stages. This composition does not allow the CNO-cycle to ensue at the beginning of the core hydrogen burning phase, as is the case for intermediate mass stars of higher metallicity. Instead, the ppchains must provide the nuclear energy for the star to survive approximately during the first $7.4 \times 10^{6} \mathrm{yr}$. However, the ppchains are far from being the main nuclear energy suppliers during the bulk of core hydrogen burning phase and, as soon as the temperature threshold for helium burning reactions is reached, the production of ${ }^{12} \mathrm{C},{ }^{14} \mathrm{~N}$ and ${ }^{16} \mathrm{O}$ in small amounts (at abundance thresholds of about $10^{-10}$ in mass) is enough for the CNO-cycle to take over as the primary energy supplier during this phase. It derives from our calculations that the entire core hydrogen burning phase lasts for about $2.1 \times 10^{7} \mathrm{yr}$, in good agreement with the result of Marigo et al. (2001), who obtained $2.2 \times 10^{7} \mathrm{yr}$.

The most important parameters relevant for a correct understanding of the structure and composition of our models during the hydrogen burning phase are shown in Table 1. For the sake of completeness we also show in Table 1 the same parameters for the rest of the evolutionary phases before carbon is ignited. The positions of each labelled model are also shown in Figs. 1 and 2, which display, respectively, the evolution of the central temperature as a function of the central density for both our zero metallicity $9 M_{\odot}$ star and that of a $Z=0.02$ star of the same mass, and the corresponding evolution in the Hertzsprung-Russell diagram for each of these stars. Models labelled as A, B and C correspond, respectively, to the beginning of the core hydrogen burning (hereafter $\mathrm{CHB}$ ), to the time at which the energy production through the CNO-cycle starts to take over that of the pp-chains and, finally, to the end 
Table 1. Characteristics of models at various points in the H-R diagram of Fig. 2.

\begin{tabular}{ccccccccccc}
\hline \hline Model & $t\left(10^{14} \mathrm{~s}\right)$ & $\log L$ & $\log T_{\text {eff }}$ & $\log R$ & $\log \rho_{\mathrm{c}}$ & $\log T_{\mathrm{c}}$ & $M_{\mathrm{He}}$ & $X_{\mathrm{c}}(\mathrm{He})$ & $M_{\mathrm{C}}$ & $X_{\mathrm{c}}(\mathrm{C})$ \\
\hline A & 0.0000 & 3.63 & 4.55 & 0.25 & 4.72 & 7.71 & 0.00 & 0.23 & 0.00 & 0.00 \\
B & 2.3545 & 3.74 & 4.61 & 0.17 & 5.16 & 7.87 & 1.90 & 0.41 & 0.00 & $1 \times 10^{-10}$ \\
C & 6.8994 & 3.95 & 4.53 & 0.44 & 5.24 & 7.93 & 1.90 & 1.00 & 0.00 & $3 \times 10^{-9}$ \\
D & 7.0477 & 4.03 & 4.50 & 0.54 & 6.63 & 8.07 & 1.90 & 0.99 & 0.00 & $5 \times 10^{-5}$ \\
E & 7.5843 & 4.10 & 4.53 & 0.55 & 6.34 & 8.22 & 2.03 & 0.48 & 0.52 & 0.45 \\
F & 8.0000 & 4.15 & 4.47 & 0.65 & 7.05 & 8.40 & 2.10 & 0.00 & 0.98 & 0.36 \\
G & 8.0520 & 4.08 & 4.14 & 1.29 & 8.80 & 8.64 & 2.15 & 0.00 & 1.05 & 0.36 \\
\hline
\end{tabular}

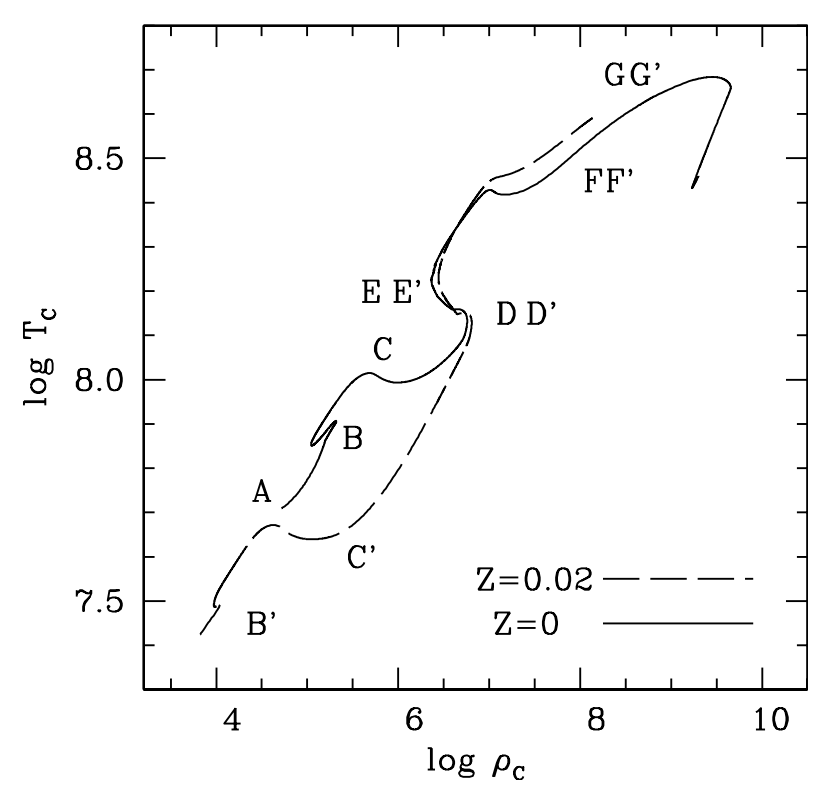

Fig. 1. Evolution in the $\log \rho_{\mathrm{c}}-\log T_{\mathrm{c}}$ plane of the zero metallicity $9 M_{\odot}$ star (solid line) and of a $Z=0.02$ star of the same mass (dashed line), up to the point where carbon is ignited.

of CHB. Models labelled as D, E and F correspond, respectively, to the beginning, to an intermediate stage, and to the end of central helium burning (hereafter $\mathrm{CHeB}$ ). Finally, model $\mathrm{G}$ corresponds to the time at which carbon is ultimately ignited.

As it can be seen in Fig. 1, during the phase in which the pp-chains are the dominant reactions - from A to B - core contraction proceeds accompanied by a smooth increase in the central temperature. However, once the CNO-cycle is activated (at B), the larger release of energy that consequently ensues temporarily stops the contraction and lets the core expand before the usual behaviour of core contraction and heating during the bulk of the CHB phase is attained. For the sake of comparison, we have also represented in Fig. 1 the evolution of the central temperature as a function of the central density for the case $Z=0.02$. As it can be seen, important differences arise. The loci in the $\log \rho-\log T$ diagram where core hydrogen burning begins and fully develops are different because hydrogen burning through the pp-chains requires higher temperatures than through the CNO-cycle to achieve the same rate of energy production. This fact also explains the positions of the associated loops in both curves. Both tracks are practically coincident at the point where the $3 \alpha$ reaction starts (D), as central

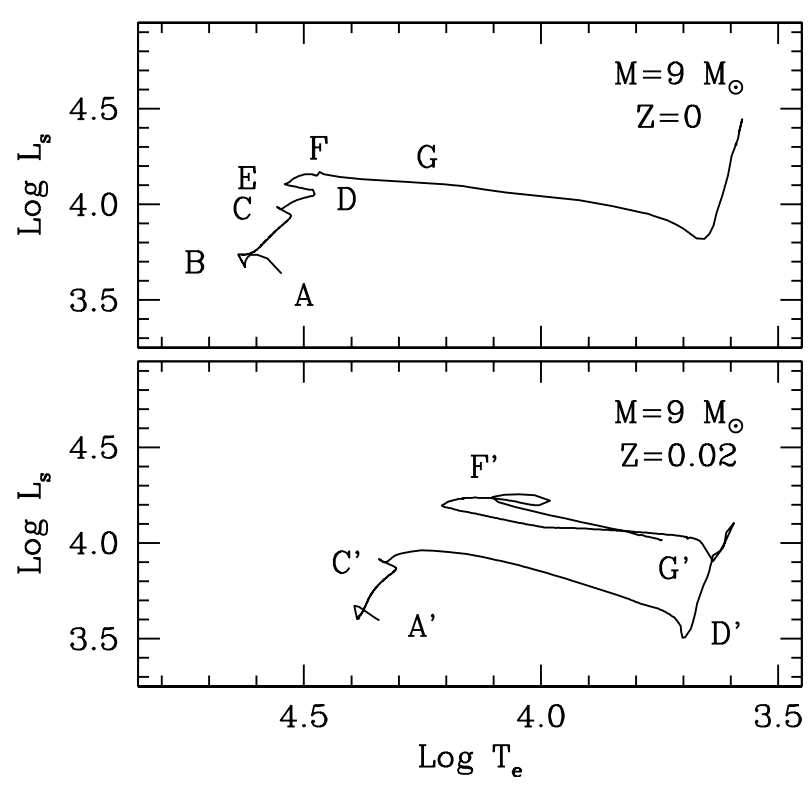

Fig. 2. Evolutionary tracks in the Hertzsprung-Russell diagram of the $9 M_{\odot}$ zero metallicity star (top panel) and of a star of $9 M_{\odot}$ with $Z=$ 0.02 (bottom panel). The labels in the $Z=0$ model correspond to the models of Table 1 . The labels in the $Z=0.02$ model correspond to equivalent evolutionary stages.

He burning is not sensitive to metallicity effects. Nevertheless the subsequent evolution takes the $Z=0$ star back to the region of higher densities.

The evolution in the Hertzsprung-Russell diagram of the zero metallicity star also presents substantial differences with respect to that of solar metallicity (see Fig. 2). The part of the core hydrogen burning phase that proceeds through the ppchains appears to occur at slightly higher effective temperatures and luminosities than those of the $Z=0.02$ case. The bulk of the core hydrogen burning phase, occuring through the CNO-cycle, leads the star to a track whose shape is very similar to that found for the $Z=0.02$ case. Nonetheless, once this process is completed, the star does not evolve yet to the low $T_{\text {eff-low }} L$ regions of the Hertzsprung-Russell diagram to reach regions close to the Hayashi track. Instead, because helium burning occurs very shortly after hydrogen is exhausted in the core, the star keeps its luminosity almost unchanged, and just a small decrease in effective temperature is observed. The ascent along the red giant branch that can be seen in Fig. 2 corresponds to the helium-shell burning phase when, as it will be shown in the next section, carbon burning has already 


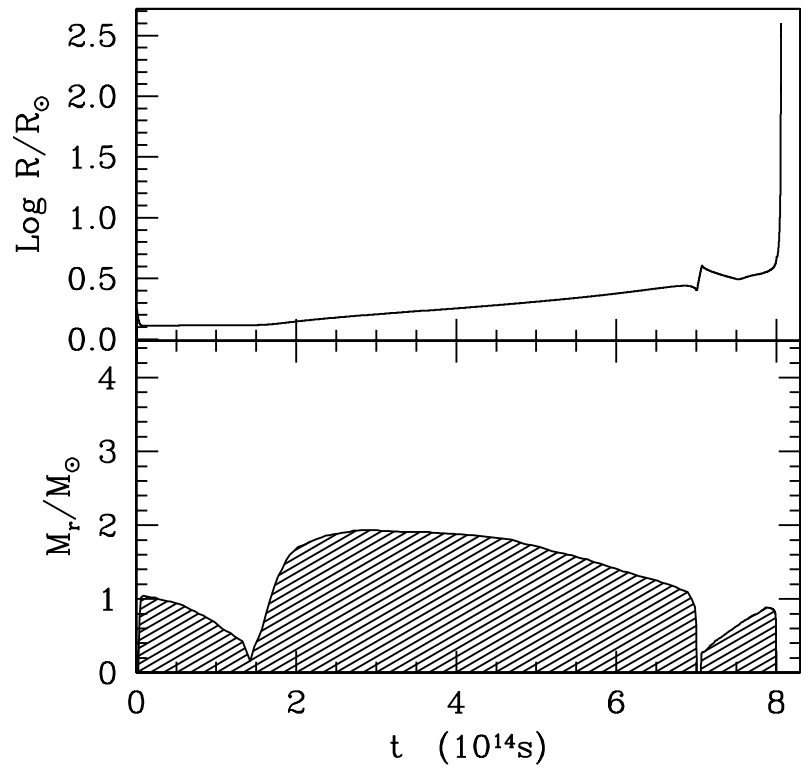

Fig. 3. Evolution of the convective regions of our model star (bottom panel) and of the radius of the star (top panel) as a function of time during the $\mathrm{CHB}$ and $\mathrm{CHeB}$ phases.

developed in the core. The former results are in agreement with, for instance, those of Marigo et al. (2001) or Chieffi et al. (2001).

The evolution of the surface radius and convective regions are shown in the top and bottom panels of Fig. 3, respectively. The part of the CHB phase during which the pp-chains dominate can be clearly distinguished from that at which the CNO-cycle takes over, as the size of the convective core is considerably smaller when the pp-chains dominate. The pronounced decrease of the size of the central convective region occuring at $t \approx \times 10^{14} \mathrm{~s}$ corresponds to a time shortly after the reactions of the $\mathrm{CNO}$ cycle set in. Moreover, during the phase of CHB dominated by the pp-chains $\left(t \approx 1.45 \times 10^{14} \mathrm{~s}\right)$ the radius of the star remains constant, and slightly increases afterwards. Note, however, that the surface radius of our model star does not increase significantly until $\mathrm{CHeB}$ is over.

The evolution of the central abundances of the main isotopes $(\mathrm{H}, \mathrm{He}, \mathrm{C}$ and $\mathrm{O})$ as a function of time up to carbon ignition are shown in Fig. 4. The step present in the evolution of the central hydrogen and helium abundances between $t \approx 1.5 \times 10^{14} \mathrm{~s}$ and $2.0 \times 10^{14} \mathrm{~s}$ is due to the competition between two effects. On the one hand, hydrogen burning tends to decrease its abundance. On the other hand, the very fast increase in the size of the central convective region that accompanies the activation of the reactions of the CNO-cycle allows fresh hydrogen to be engulfed and, eventually, keeps its abundance constant. The slopes of $X\left({ }^{1} \mathrm{H}\right)$ and $X\left({ }^{4} \mathrm{He}\right)$ as a function of time are also different during the phase in which the pp-chains dominate and during the CNO-cycle burning phase. At the end of the helium burning phase the He-exhausted core has a mass of about $1.1 M_{\odot}$, and is composed of carbon $\left(X\left({ }^{12} \mathrm{C}\right) \simeq 0.34\right)$ and oxygen $\left(X\left({ }^{16} \mathrm{O}\right) \simeq 0.65\right)$. These abundances differ from those obtained by Marigo (2001), whose calculations yielded $X\left({ }^{12} \mathrm{C}\right) / X\left({ }^{16} \mathrm{O}\right)=0.658$. Also the size of the He-exhausted core shown here is smaller than that obtained

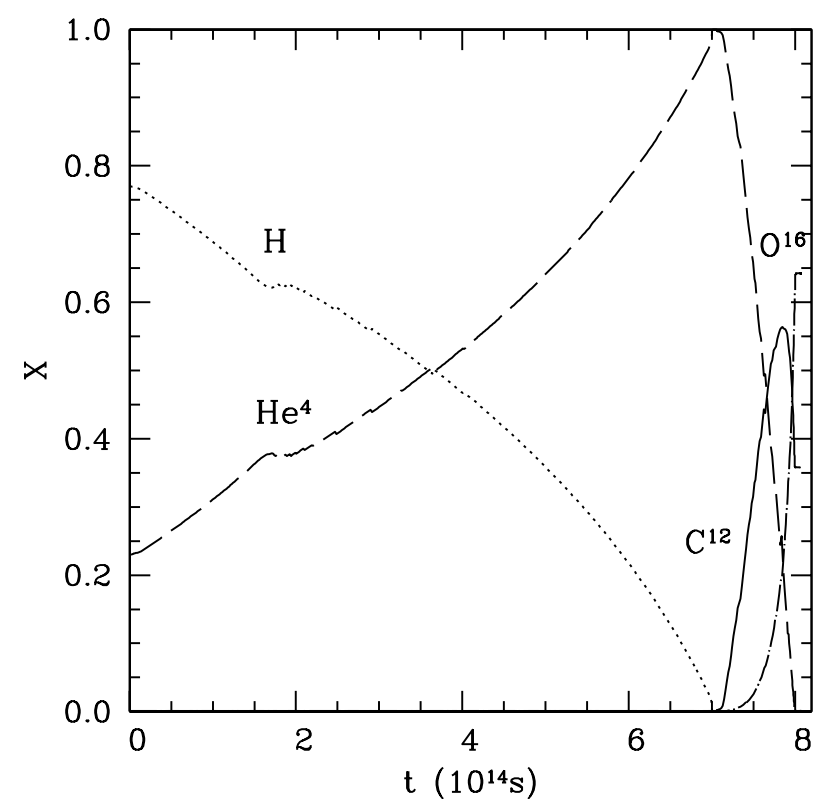

Fig. 4. Evolution of the central abundances of the $9 M_{\odot}$ primary until the beginning of carbon burning.

by Marigo (2001), who obtained a $1.29 M_{\odot}$ He-exhausted core. The differences in the $\mathrm{CO}$ core masses and abundance ratios are probably a consequence of the different treatments adopted for convection. Marigo (2001) considered a moderate amount of overshooting in their calculations, whereas we are not using overshooting at all.

\section{The carbon burning phase}

Figure 5 shows the temporal evolution of the main structural quantities just after the $\mathrm{CHeB}$ phase and during the bulk of the carbon phase. In particular, in the top panel of Fig. 6 the helium and carbon luminosities are shown (solid and dashed lines, respectively). The central panel of Fig. 5 shows the variations of the radius and the luminosity. Finally, the bottom panel of Fig. 5 shows the behavior of the convective regions during this phase. The vertical thin line in all three panels indicates when the carbon burning luminosity is $10 \%$ of the helium burning luminosity, corresponding to $t=8.0562 \times 10^{14} \mathrm{~s}$. Regions to the left of this line correspond to the phase in which the helium burning shell is the dominant nuclear energy source, whereas regions to the right of this line correspond to phases in which carbon burning is significant.

Once the $\mathrm{CHeB}$ phase has been completed, the He-burning reactions are established in a shell and, simultaneously, the star begins an excursion to the red part of the HertzsprungRussell diagram at slightly decreasing luminosities, as shown in Fig. 2 and in the central panel of Fig. 5. At this point $\left(t=8.0571 \times 10^{14} \mathrm{~s}\right)$ the surface radius of the star has increased from an initial value of about $20 R_{\odot}$ up to roughly $320 R_{\odot}$. This overall expansion is accompanied by the formation of a convective envelope, whose inner edge is able to penetrate relatively deep inside the star - see the bottom panel of Fig. 5. However, the bottom of the convective envelope stops its advance at $t=8.0571 \times 10^{14} \mathrm{~s}$, before it can reach the regions 


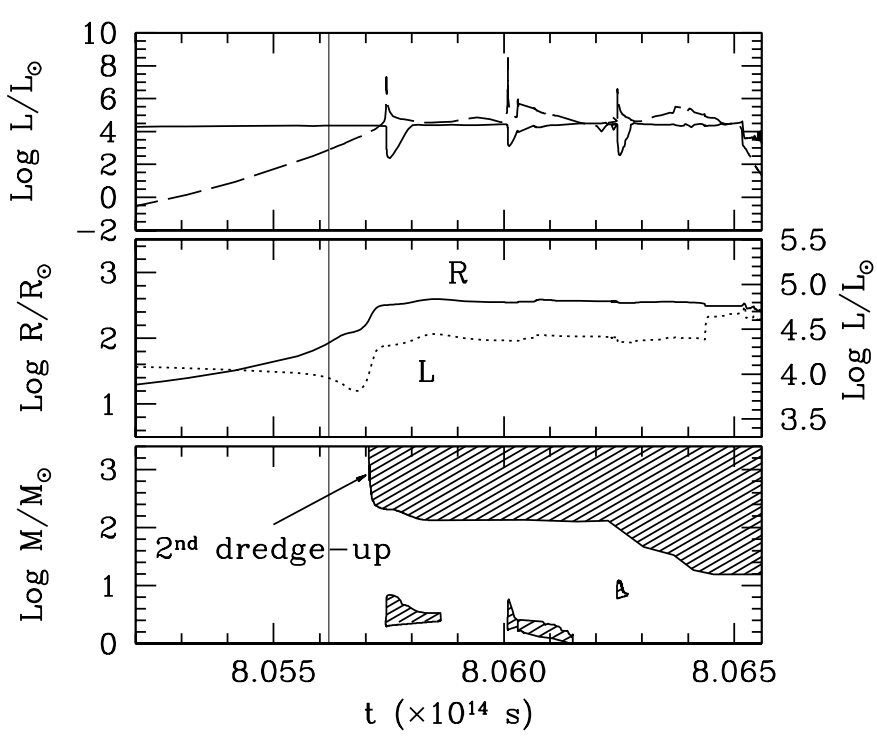

Fig. 5. Temporal evolution of the main structural parameters during the bulk of the carbon burning phase. In the top panel the luminosity associated to helium burning (solid line) and carbon burning (dashed line) are shown. The central panel displays the evolution of the stellar surface radius (solid line) and of the luminosity (dashed line). In the bottom panel the evolution of the convective regions is represented. See text for details.

of the star that have been previously enriched in products of the $\mathrm{H}$ nuclear reactions. During this process, when the maximum extent inwards of the base of the convective envelope (BCE from now on) is reached $\left(M_{\mathrm{BCE}}=2.15 M_{\odot}\right)$, the surface abundances (by mass) of carbon, nitrogen and oxygen are, respectively, $X\left({ }^{12} \mathrm{C}\right)=8.74 \times 10^{-12}, X\left({ }^{14} \mathrm{~N}\right)=6.61 \times 10^{-10}$ and $X\left({ }^{16} \mathrm{O}\right)=7.84 \times 10^{-12}$. Therefore, during the early phase of this (so-called) second dredge-up, no significant changes can be observed in the surface composition of the star. It must be noted that, strictly speaking, we do not find a first dredge-up during hydrogen shell burning, as it is the case of higher metallicity objects, and consequently it must be stressed that calling second dredge-up this penetration of the convective envelope is just a matter of keeping consistency with the nomenclature used for stars of higher metallicity.

Carbon burning begins just before the second dredge-up, at $t=8.0534 \times 10^{14} \mathrm{~s}$, while the star is still climbing the red giant branch. Note that - as previously mentioned - at time $t=8.0571 \times 10^{14} \mathrm{~s}$, the surface radius stops increasing and the convective envelope temporarily halts its inner advance. This is due to the increasing energy flux released by the vigorous carbon burning reactions occurring deep inside the Heexhausted core. As a consequence of the ignition of carbon the $\mathrm{He}$-exhausted core stops contracting and releasing gravitational energy and, consequently, the BCE stops moving inwards. Our model star behaves in many senses in a way similar to that of solar metallicity stars of slightly higher mass. In particular, carbon is burnt off-center in conditions of partial degeneracy in a series of three consecutive flashes - top panel of Fig. 5. The first flash starts at $M_{\mathrm{r}} \simeq 0.33 M_{\odot}-$ bottom panel of Fig. 5 and the carbon burning luminosity reaches values as high as $L_{\mathrm{C}} \simeq 10^{8} L_{\odot}$.

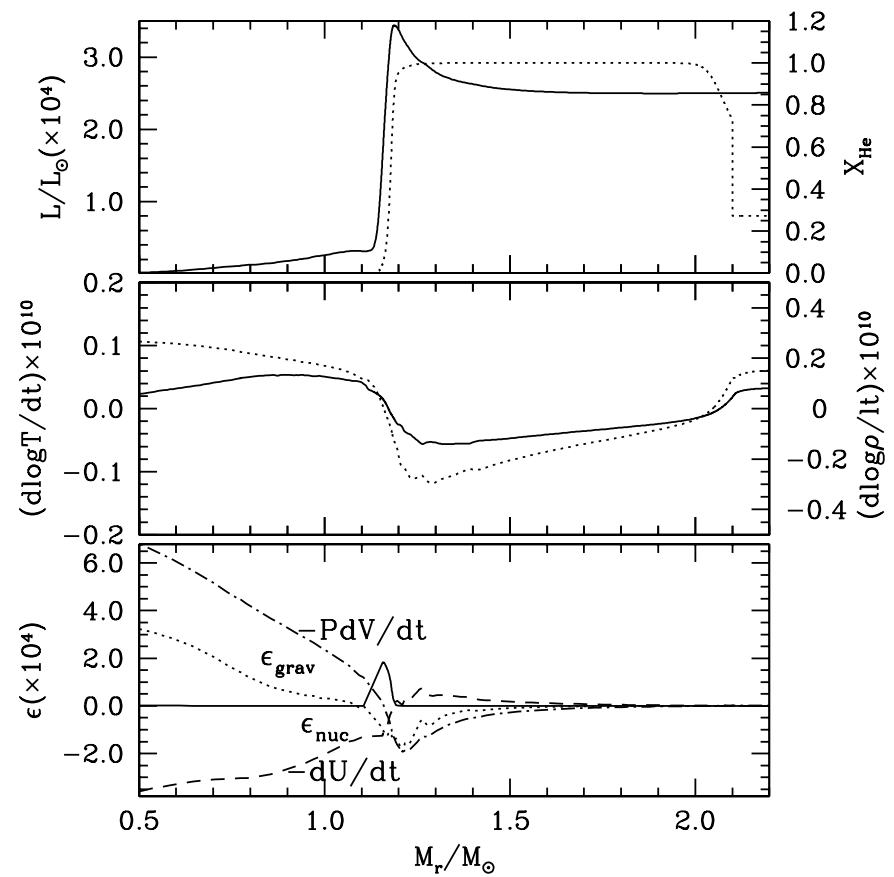

Fig. 6. Relevant structural and dynamical quantities for a model at time $t=8.0616 \times 10^{14} \mathrm{~s}$, just before the second advance of the convective envelope, in a region extending from below the helium burning shell until the base of the convective envelope. Upper panel: velocity (solid line) and helium profile (dotted line). Middle panel: time variation of the temperature (solid line) and density (dotted line). Lower panel: energy release rates (nuclear and gravothermal) along with the time derivative of the internal energy and the work of expansion. The nuclear energy release rate has been divided by 10 in order to fit into the scale.

Fast injections of energy at very high rates and in relatively small regions of the stellar interior induce high temperature gradients and, consequently, several inner convective regions associated to each flash develop - see the bottom panel of Fig. 5. Part of the energy released in these flashes is transformed into work of expansion that changes the physical structure of the layers where helium burning is still active. The expansion and cooling of these layers lead to an effective decrease in helium luminosity, that recovers once the carbon flash is finished, very much in the same way solar metallicity stars of the same mass do. Nevertheless these dramatic changes affect mainly the innermost regions of the star, and they have a rather limited effect on the structure of the outer envelope. In fact, the surface radius and luminosity remain almost constant, similarly to what happens to the $Z=0.02$ stars of $10 M_{\odot}$, $10.5 M_{\odot}$ and $11 M_{\odot}$ discussed in Ritossa et al. (1995), Iben et al. (1997) and García-Berro et al. (1999), and in sharp contrast with the behavior of the $9 M_{\odot}$ star of solar metallicity discussed in García-Berro et al. (1997). The reason of this behavior is that in the case of the solar metallicity star carbon is ignited at $M_{\mathrm{r}} \simeq 0.46 M_{\odot}$, much closer to the helium-carbon discontinuity than in the case of our $Z=0$ model. Hence, for the solar metallicity star the carbon flashes are felt all the way to the base of the convective envelope much more conspicuously than in the $Z=0$ model star. 
As a consequence of the second flash, at time $t=8.06021 \times$ $10^{14} \mathrm{~s}$, two important facts occur. First, the carbon burning front reaches the center of the star. It is worth noting at this point that although the main carbon burning phase (corresponding to the second flash) starts indeed as a flash it proceeds as a flame, as discussed in depth in García-Berro et al. (1997). Second, once the center is reached, a new inward advance of the convective envelope takes place (see Fig. 5). During the first phase of the dredge-up episode, the base of the convective envelope reached a mass coordinate of $2.15 M_{\odot}$, at which it stabilized for most of the carbon burning phase. After the carbon burning front reaches the center the BCE moves down to the regions located deeper in the star. As it can be seen in Fig. 5, this penetration of the convective envelope occurs in two stages, the first one reaching $M_{\mathrm{r}}=1.74 M_{\odot}$, where it stops temporarily, and the second one reaching $M_{\mathrm{r}}=1.20 M_{\odot}$. In fact the BCE gets deep enough to reach regions of the star where hydrogen and helium burning have efficiently proceeded and, therefore, the elements of the CNO-cycle and the $\alpha$-products are dredged-up to the stellar surface. The final surface abundances of carbon, oxygen and nitrogen after this additional dredge-up episode are, respectively, $X\left({ }^{12} \mathrm{C}\right)=2.04 \times 10^{-4}, X\left({ }^{14} \mathrm{~N}\right)=2.41 \times 10^{-6}$ and $X\left({ }^{16} \mathrm{O}\right)=3.47 \times 10^{-6}$.

As already discussed, the first inwards advance of the BCE took place during the helium burning shell phase. To reach a better understanding on how the dredge-up process occurs, we have represented in Figs. 6 and 7 the luminosity and helium abundance profiles (upper panel), the relative variation of the temperature and density with time (middle panel), and the different rates of energy generation and absorption (bottom panel). Figure 6 corresponds to a time $t=8.0616 \times 10^{14} \mathrm{~s}$, just after the carbon burning front has reached the center of the star. As it can be seen, the generation of nuclear energy only occurs at the helium burning shell (HeBS), which is located at $M_{\mathrm{r}} \simeq 1.1926 M_{\odot}$. This energy is partially absorbed in the region above the HeBS (of thickness $0.2 M_{\odot}$ ) and produces a slight expansion of these layers. Between this zone and the BCE, the structure variables $T$ and $\rho$ and the luminosity remain approximately constant.

Figure 7 shows the same quantities as in Fig. 8, but at a time $t=8.0629 \times 10^{14} \mathrm{~s}$, during the second inner advance of the envelope. The situation is now very different since at this moment a new mild carbon flash is occuring relatively close to the carbon-helium discontinuity. The region in which carbon is burnt is located in the most external regions of the partially degenerate core, at only $0.3 M_{\odot}$ below the carbon-helium discontinuity. Consequently, the layers between the carbon-helium discontinuity (which is located at $M_{\mathrm{r}} \simeq 1.1936 M_{\odot}$ ) and the BCE start expanding and cooling. Hence, the radiative gradient in this region increases over the adiabatic gradient and, consequently, the BCE advances towards the center. However, and opposite to what occured in the previous carbon flashes, the third flash is not strong enough to halt the expansion and cooling of the region between the HeBS and the BCE, and therefore, the convective zone keeps advancing.

As it has been already mentioned, after the second carbon flash, a third flash of smaller strength occurs. The maximum luminosity is in this case of about $L_{\mathrm{C}} \approx 3.0 \times 10^{6} L_{\odot}$. Moreover,

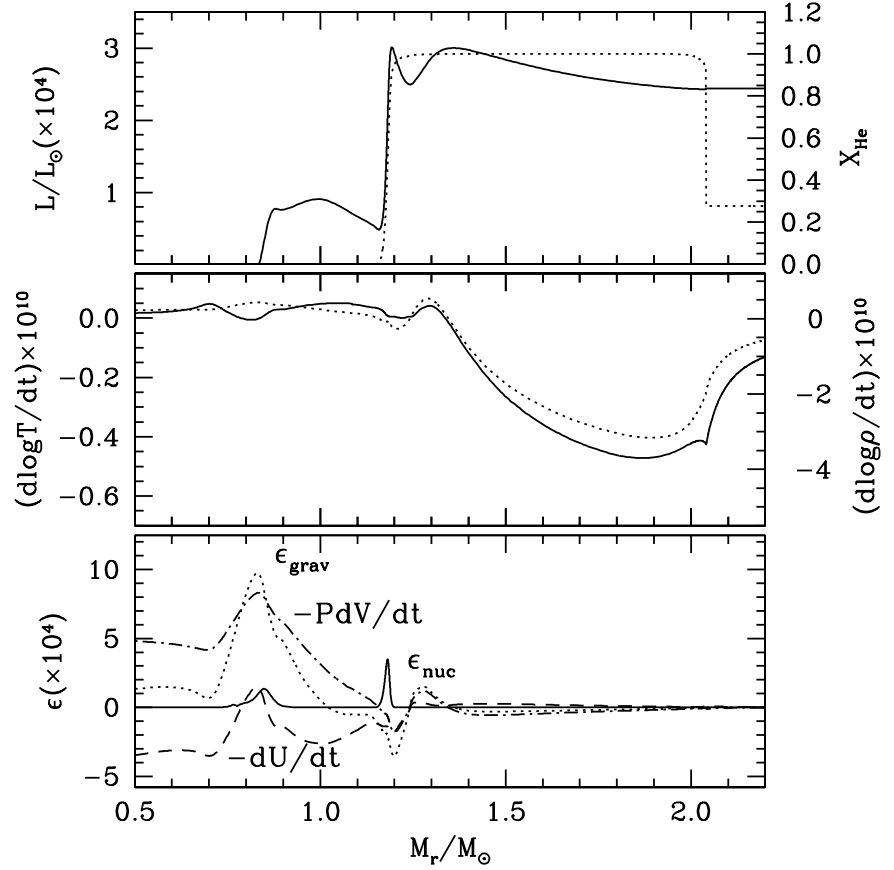

Fig. 7. Same as Fig. 8, but for time $t=8.0629 \times 10^{14} \mathrm{~s}$, during the second inner advance of the envelope.

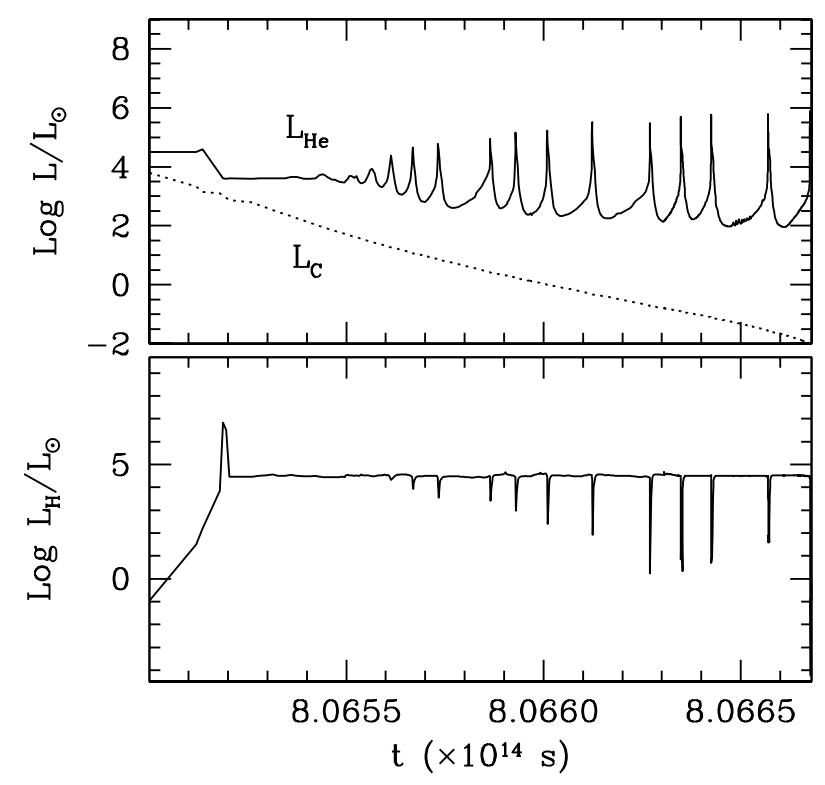

Fig. 8. Temporal evolution of the first 10 thermal pulses. The upper panel displays the luminosity associated to helium burning, whereas lower panel represents the evolution of the luminosity associated with hydrogen burning.

in this case the associated convective zone develops in a region of the core which is closer to the BCE and, hence, mildly degenerate. After this flash, the remaining carbon burns out more quietly. The subsequent small decrease in the surface radius of the star (see the central panel of Fig. 5) and the overall heating of its envelope, as well as the presence of CNO elements in regions just below the base of the convective envelope allows a reignition of the hydrogen burning shell. The conditions of degeneracy allow for a hydrogen shell flash - see Fig. 8. This flash 


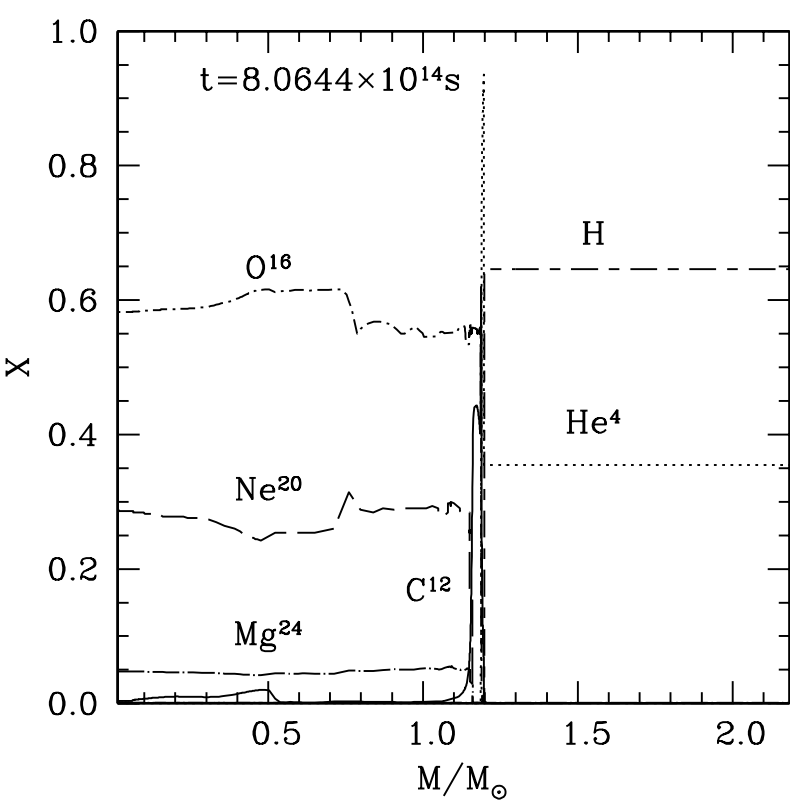

Fig. 9. Abundance profiles of the core at the end of the carbon burning phase.

has a magnitude of $L_{\mathrm{H}} \simeq 10^{7} L_{\odot}$, lasts for $60 \mathrm{yr}$, and occurs at $t=8.065176 \times 10^{14} \mathrm{~s}$ in a region $\left(M_{\mathrm{r}} \simeq 1.192763 M_{\odot}\right)$ just below the BCE (which is located at $M_{\mathrm{r}} \simeq 1.192843 M_{\odot}$ ), and very close to the locations of the almost extinguished carbon burning shell at $M_{\mathrm{r}} \simeq 1.178429 M_{\odot}$ and of the helium burning shell, at $M_{\mathrm{r}} \simeq 1.191962 M_{\odot}$. The associated convective region is very small $\left(\sim 10^{-5} M_{\odot}\right)$ and rapidly merges with the convective envelope. At this time the carbon luminosity is $L_{\mathrm{C}} \simeq 1.3819 \times 10^{3} L_{\odot}$ and the helium luminosity is $L_{\mathrm{He}} \simeq 1.1071 \times 10^{4} L_{\odot}$. This flash is a direct consequence of the the compression of the layers just below the $\mathrm{BCE}$ which, in turn, is a consequence of the extinction of the carbon burning shell. However, after the resurrection of the hydrogen burning shell degeneracy is quickly removed due to the expansion and cooling which derive from the fast injection of energy in the layers involved. Afterwards, hydrogen burning is not quenched, but instead it proceeds at approximately constant luminosity of about $L_{\mathrm{H}} \approx 3.0 \times 10^{4} L_{\odot}$. The reactivation of the hydrogen burning shell increases the mass of the He buffer and, consequently, marks the beginning of the alternance of hydrogen and helium shell flashes, which interchange themselves as main nuclear energy suppliers of the star. Therefore, our model star begins the thermally pulsing SuperAsymptotic Giant Branch, which will be described in detail in Sect. 4. The term Super-AGB stars was coined by Ritossa et al. (1995) to refer to AGB stars with ONe cores. In this regard it is worth having a look at the abundance profiles of the inner $\mathrm{ONe}$ core. The core abundances after the main carbon-burning phase are shown in Fig. 9. The abundance pattern does not present striking differences with respect to the solar metallicity corresponding core. However, the core of the zero metallicity star is slightly overabundant in ${ }^{20} \mathrm{Ne}$ with respect to the solar metallicity one $-X\left({ }^{20} \mathrm{Ne}\right)=0.28$ versus $X\left({ }^{20} \mathrm{Ne}\right)=0.24$ - and somewhat underabundant in oxygen. Finally, the final mass of the C-exhausted core in the $Z=0$ case is $M_{\mathrm{c}}=1.15 M_{\odot}, 0.09 M_{\odot}$ larger than that of its $Z=0.02$ counterpart.

\section{The thermal pulses}

Figure 8 shows the temporal evolution of the hydrogen luminosity. We have computed 14 thermal pulses of which only the first 10 thermal pulses are shown in Fig. 8. After the first hydrogen flash, a quiet phase during which helium accumulates follows. During this phase the luminosity of the hydrogen burning shell remains nearly constant $\left(L_{\mathrm{H}} \simeq 3.0 \times 10^{4} L_{\odot}\right)$ and the helium burning shell is still active, opposite to what was found by Ritossa et al. (1995) for a solar metallicity star of $10 M_{\odot}$. However, helium is radiatively burnt at the base of the helium buffer at a rate smaller than that of hydrogen at the base of its burning shell, the net result being that the mass of the helium buffer increases. After some time, the helium buffer reaches the critical mass and, consequently, a series of thermal pulses begins. The first few pulses are rather weak, as usually found for this kind of stars - Ritossa et al. (1995) and García-Berro et al. (1997) found some mini-pulses, which are equivalent to what is found here - but, as time advances, the amplitude of the pulses increases. Note as well that the inter-pulse time interval also varies from one cycle to the next. By the end of the calculations shown here the amplitude of the pulses remained almost constant with an approximate value of $L_{\mathrm{He}}^{\max } \simeq 6.0 \times 10^{5} L_{\odot}$ for the maximum luminosity and of about $L_{\mathrm{He}}^{\min } \simeq 10^{2} L_{\odot}$ for the minimum luminosity, whereas the inter-pulse time interval did not, ranging between $\tau \approx 250 \mathrm{yr}$ and $400 \mathrm{yr}$. For the sake of comparison, recall that García-Berro et al. (1997) found a maximum luminosity of about $6.0 \times 10^{4} L_{\odot}$ for their solar metallicity $9 M_{\odot}$ model star, and a much more regular inter-pulse time interval of $400 \mathrm{yr}$, whereas Ritossa et al. (1995) found a maximum luminosity of about $2.7 \times 10^{6} L_{\odot}$ and an inter-pulse period of roughly $200 \mathrm{yr}$ for their solar metallicity $10 M_{\odot}$ model. Thus the thermal pulses of our $Z=0$ model star of $9 M_{\odot}$ are similar to those of a model star of slightly larger mass of solar metallicity, as we have also found for other characteristics (coupling of the interior and the envelope during the carbon burning phase, size of the core, chemical composition of the He-exhausted core ...) of our model star.

The evolution of the temperature at the base of the convective envelope during the pulses is also midway between those of $10 M_{\odot}$ and $9 M_{\odot}$ model stars of solar composition. During the mini-pulses it adopts a typical value of $T_{\mathrm{BCE}} \simeq 3 \times 10^{7} \mathrm{~K}$ monotonically increasing to $T_{\mathrm{BCE}} \simeq 8 \times 10^{7} \mathrm{~K}$ up to the eighth pulse. From this thermal pulse on, the temperature at the base of the convective envelope remains roughly constant and enough to transform part of the freshly synthesized CNO elements into ${ }^{25} \mathrm{Mg}$ and neutrons. As to the existence of a third dredge-up, we have not detected such a process after following 14 helium flashes. However, dredge-up processes are strongly dependent on the treatment of convection, and the fact that we have not introduced any overshooting in our calculations might have prevented the development of an additional dredge-up.

The thermally pulsing AGB phase of low and intermediate mass primordial stars has been thoroughly studied by Siess et al. (2002). They found two different mechanisms allowing for the thermal pulses. For objects between 1 and $5 M_{\odot}$, a convective instability develops at the He-H discontinuity causing the ingestion of carbon and, consequently, 
reactivating the CNO-cycle. Instead, for the $7 M_{\odot}$ object it is a previous dredge-up phase which allows the enrichment in $\mathrm{CNO}$ products and the subsequent behaviour as a normal thermally pulsing AGB star. Our $9 M_{\odot}$ model star behaves very much in the same way as the $7 M_{\odot}$ star described by Siess et al. (2002). Both our results and those of Siess et al. (2002) contradict the pioneering work of Fujimoto et al. (1984). These authors found that there was a threshold value at $M_{*}=0.73 M_{\odot}$ such that stars whose core mass was lower than $M_{*}$ experienced thermal pulses, but stars with a core more massive than this threshold value could only experience thermal pulses if their metallicity was above $Z \approx 10^{-7}$. However, as already shown by Siess et al. (2002) and here, the pollution in CNO-elements caused by convection in the He-H discontinuity zone or caused by previous dredge-up phases makes this requirement on initial metallicity unnecessary.

Finally, it is interesting to have a look at the surface ratios of carbon, nitrogen and oxygen. For the solar metallicity stars of masses 9 and $10 M_{\odot}$ studied before in Ritossa et al. (1995) and García-Berro et al. (1997) these ratios are respectively $(\mathrm{C}: \mathrm{N}: \mathrm{O})=(1.63: 3.63: 6.93)$ and $(2.35: 4.25: 6.26)$ after the second dredge-up, and the total metallicities are in both cases $Z \simeq 0.012$ at this moment. In sharp contrast the initially $Z=0$ star of $9 M_{\odot}$ studied here presents $(\mathrm{C}: \mathrm{N}: \mathrm{O})=(2.03: 0.02: 0.03)$ for a total metallicity of $Z=2.1 \times 10^{-4}$. Thus, observationally our model star would look like a $\mathrm{C}$-enhanced metal-poor star.

\section{Mass loss and evolution to white dwarf}

In Fig. 10 the latest stages of the evolution in the HertzprungRussell diagram of the zero metallicity $9 M_{\odot}$ star are presented. The most important evolutionary stages have been highlighted along the evolutionary track. In particular, the beginning of the carbon burning phase has been marked with a circle, whereas the diamond shows the end of the thermally pulsing SuperAGB (TP-SAGB) phase. After the eleventh thermal pulse, when the surface radius is $R_{\mathrm{S}} \simeq 320 R_{\odot}$ and the surface luminosity is $L_{\mathrm{s}} \simeq 6.5 \times 10^{3} L_{\odot}$, we have assumed, somehow arbitrarily, that a mass-loss episode ensues. Before the eleventh pulse no mass-loss was assumed to occur. This massloss episode is clearly marked in Fig. 10 using a thick solid line.

As occurs for solar metallicity objects of analogous ZAMS mass, the mass-loss episode is plagued with many uncertainties, as no definite mass-loss rates are available for low metallicity stars, and there is not even a well studied mechanism responsible for these mass-loss episodes - see, however, Marshall et al. (2004). Stellar winds for the case of single stars as well as Roche lobe overflow for the case of binaries are likely to play important roles, as it usually occurs for solar metallicity stars of analogous masses. With the values of the luminosity and the radius at the end of the eleventh thermal pulse the mass-loss rates due to stellar winds would be significant for a solar metallicity star of the order of $10^{-6} M_{\odot} \mathrm{yr}^{-1}$, according to Reimers (1975) and Nieuwenhuijzen \& de Jager (1990). We recall that the convective envelope of our model star presents a significant enrichment in metals $\left(Z=2.1 \times 10^{-4}\right)$ which, nevertheless, is smaller than that of a solar metallicity star of the same

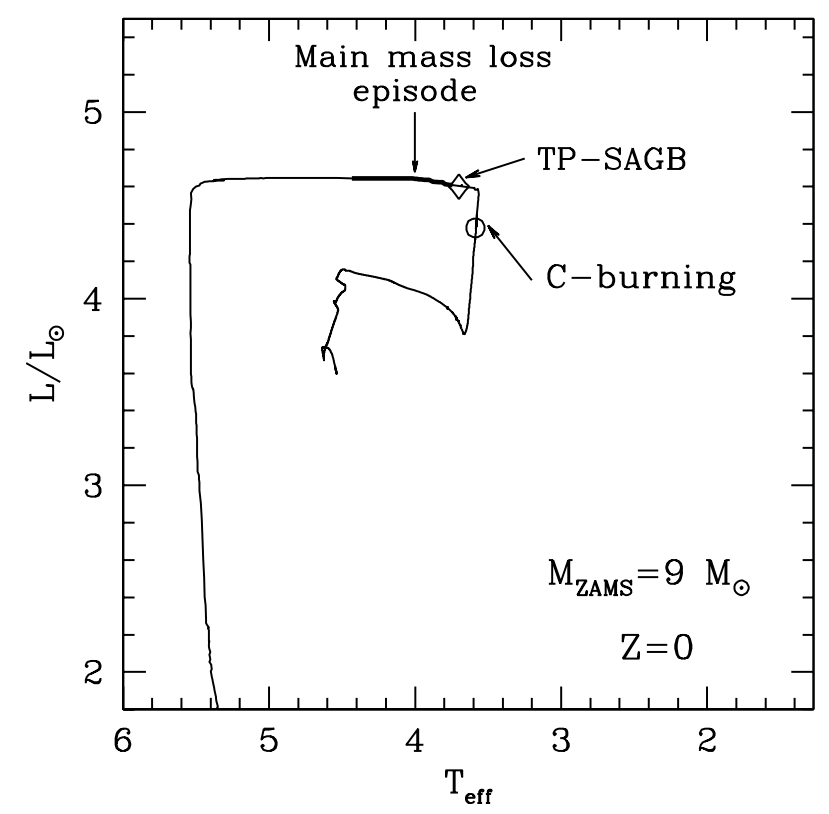

Fig. 10. Evolution in the Hertzsprung-Russell diagram of the $9 M_{\odot}$ star of zero metallicity (solid line) and of the $Z=0.02$ star of the same mass (dotted line). The circle corresponds to the beginning of the $\mathrm{C}$ burning phase and the diamond corresponds to the end of the thermally pulsing Super-AGB (TP-SAGB) phase. The thick line at the early horizontal track corresponds to the mass loss episode. See text for further details.

mass. Consequently, to a first approximation we can consider the mass-loss rates of Nieuwenhuijzen \& de Jager (1990) as representatives of the real mass-loss rate. Additionally, the extension of the mass-loss episode must also be taken with some caution and, consequently, the final limit of the thick line in Fig. 10 must also be considered as an approximate value.

Keeping this in mind, we have performed different sets of calculations of the latest evolutionary stages, to cover the effects of mass loss with rates between $\sim 10^{-7} M_{\odot} \mathrm{yr}^{-1}$ and $\sim 10^{-4} M_{\odot} \mathrm{yr}^{-1}$, which bracket the representative value derived above. The evolutionary sequences computed with low rates would describe the effects of mass loss due to stellar winds, and the sets of evolutionary sequences computed with higher rates (between $10^{-5} M_{\odot} \mathrm{yr}^{-1}$ and $\sim 10^{-4} M_{\odot} \mathrm{yr}^{-1}$ ) might describe mass loss due to Roche lobe overflow in a binary system. The mass-loss rate used for the evolutionary sequence shown in Fig. 10 is $\dot{M}=3.0 \times 10^{-6} M_{\odot} \mathrm{yr}^{-1}$. Finally, it is important to say that, in all the cases studied here we have encountered some computational difficulties at the latest evolutionary stages - as is usually found for AGB stars with carbon-oxygen cores with masses smaller than those considered here - but no significant differences regarding the final size and composition of the remnant core have been found.

Finally, Fig. 11 shows the chemical profiles of the remnant of the zero metallicity $9 M_{\odot}$ star. The mass of the core is $\sim 1.19 M_{\odot}$. If we compare with the central regions of the model presented in Fig. 9, corresponding to a time shortly after the end of the carbon burning phase, it can be seen that some sharp features in the abundance profiles after the bulk of the carbon burning phase have been smeared out due to the Rayleigh-Taylor 


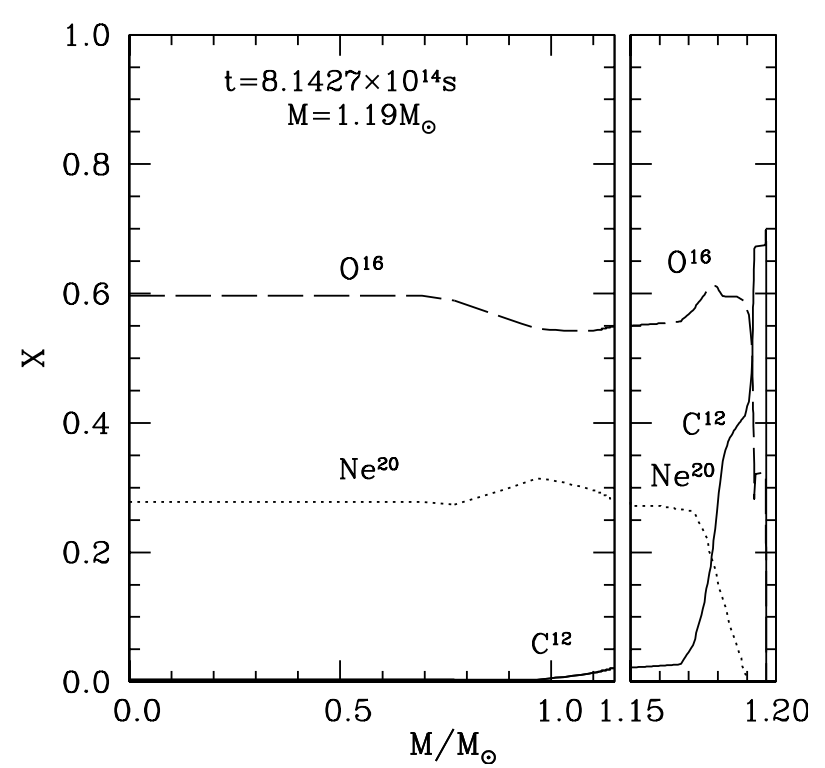

Fig. 11. Main isotope abundance profile of the zero metallicity $9 M_{\odot}$ remnant star.

instabilities (Salaris et al. 1998) that occur in the subsequent evolution of the star, but neither the size nor the general composition of the core have substantially changed. The main isotopes present in the core are ${ }^{16} \mathrm{O}$ and ${ }^{20} \mathrm{Ne}$, with mass abundances $\sim 0.59$ and $\sim 0.28$, respectively. As in the case of the solar metallicity stars of similar mass (Ritossa et al. 1995; Gil-Pons \& García-Berro 2001), some small amount of unburnt carbon is present (with a maximum mass abundance of $\sim 0.02$ before the Rayleigh-Taylor instabilities mix it through out the inner regions of the core). Finally, in the $Z=0$ case no significant amounts of ${ }^{24} \mathrm{Mg}$ nor ${ }^{23} \mathrm{Na}$ have been found.

\section{Summary and conclusions}

We have computed the evolution of a zero metallicity $9 M_{\odot}$ star from the zero age main sequence until the formation of an $\mathrm{ONe}$ white dwarf, through core carbon burning and the TP-SAGB phase. Previous works on zero metallicity intermediate mass evolution have recently been made by Marigo et al. (2001), Marigo (2002), Heger \& Woosley (2002) and Chieffi et al. (2001), but these authors did not follow carbon ignition in the degenerate core. Hence, this is the first calculation in which the carbon burning phase and the thermal pulses of a heavyweight intermediate mass primordial star have been followed self-consistently.

Our results compare favorably with those of Marigo et al. (2001) and Marigo (2002) for a $9 M_{\odot}$ model star. In particular, the time scales for hydrogen burning are notably similar in both calculations. We do not obtain such a close agreement for the final size of the $\mathrm{CO}$ cores, since our calculations yield $\mathrm{a} \simeq 1.1 M_{\odot} \mathrm{CO}$ core, whereas they obtain a CO core of $1.28 M_{\odot}$. However, it should be noted that even though we both use the OPAL radiative opacities (Rogers \& Iglesias 1992; Iglesias \& Rogers 1993), and the conductive opacities of Hubbard \& Lampe (1969), our respective equations of state are not exactly the same. Concerning the nuclear reaction rates, we both are using basically the reaction rates of Caughlan \& Fowler (1988), but Marigo et al. (2001) increased the rate for the controversial ${ }^{12} \mathrm{C}(\alpha, \gamma){ }^{16} \mathrm{O}$ reaction by a factor of 1.7 (Weaver \& Woosley 1993), whereas we did not. Finally, the factor that is more likely to ultimately determine the differences in the sizes of our respective $\mathrm{CO}$ cores is probably the treatment of convection. Marigo et al. (2001) used moderate convective core overshooting, whereas we did not use it at all.

We obtain a partially degenerate $\mathrm{CO}$ core in which carbon is ignited off-center. Carbon burning proceeds through a series of flashes which generates inner convective regions, as is found for stars of similar mass of solar metallicity. Regarding convection, a calibration of the parameters of the Mixing Length Theory (and in particular of the amount of overshooting) can only be made for the case of solar metallicity by comparing with the abundant observational material. This procedure provides reliability to the MLT results, but the problem in the case of $Z=0$ stars is the lack of observations and, therefore, of an appropriate calibration. Given the lack of observational material we have preferred to stick as close as possible to our previous calculations (Gil-Pons \& García-Berro 2001, 2002) in which no overshooting was adopted. Although the evolution during carbon burning for the $Z=0$ model star is very similar to that of solar metallicity, it is important to note one difference between the two models, namely, that the envelope of the $Z=0$ star behaves as if it were more decoupled from the core than its solar metallicity counterpart. Hence, its structure is not significantly changed by the release of energy that occurs at the innermost regions of the star. Once carbon is depleted in most of the central regions of the He-exhausted core we find that the base of the convective envelope advances inwards and dredges up nuclear processed elements. Finally, the hydrogen burning shell is reactivated and a thermally pulsing Super-AGB (an AGB star with an ONe core) is formed. We have followed the first 11 pulses and we have found that their characteristics are midway between those computed for solar metallicity stars of 9 and $10 M_{\odot}$.

Arbitrarily we have chosen mass loss to begin at $t=$ $8.066307 \times 10^{14} \mathrm{~s}$, when the eleventh thermal pulse is already over and the surface radius is approximately $320 R_{\odot}$. At this stage, the dredge-up process following core helium exhaustion has already taken place. We have not delved into the causes of mass loss, which might correspond either to stellar winds or to Roche lobe overflow (in binary systems). Instead, because no parametrization of these processes has been made up to now, we have computed a series of sequences with mass loss rates between $10^{-7} M_{\odot} \mathrm{yr}^{-1}$ and $\sim 10^{-4} M_{\odot} \mathrm{yr}^{-1}$. No significant differences have been found regarding the mass of the remnant and its composition. We obtain a ultramassive helium-rich $1.19 M_{\odot}$ white dwarf formed by a $1.17 M_{\odot}$ oxygen-neon core and a $0.02 M_{\odot}$ carbon-oxygen buffer. Such ultramassive white dwarfs have been detected by Finley et al. (1997) in a spectroscopic survey of DA white dwarfs hotter than $25000 \mathrm{~K}$, and by Dupuis et al. (2002) in the EUVE and ROSAT surveys. More recently, Liebert et al. (2004) have found a tail of massive white dwarfs in the mass distribution of white dwarfs in the Palomar Green Survey. Moreover, these authors have pointed out that of 28 white dwarfs all but nine are within $\pm 30^{\circ}$ of the Galactic 
plane, thus suggesting that there is an association between these white dwarfs and that of $\mathrm{B}$ and $\mathrm{O}$ stars. Additionally, they noticed that the ultramassive component of the white dwarf mass distribution has small cooling ages, suggesting massive progenitors. In summary, all these surveys have provided a handful of ultramassive white dwarfs and the derived mass distributions show a high mass shoulder with a peak centered at $\sim 0.9 M_{\odot}$ that provides an excess of white dwarfs with masses between 1.0 and $1.2 M_{\odot}$. Since the theoretical mass distributions using a steady initial-to-final mass relation do not predict such behavior, Weidemann (2000) was the first to ascribe this behavior to an unsteady initial-to-final mass relation which separates white dwarfs with carbon-oxygen cores from those with oxygen-neon cores like the one obtained in the present work. Although the unsteady behavior has also been ascribed to binary evolution, the matter still remains open, as recent Smoothed Particle Hydrodynamics simulations (Guerrero et al. 2004) do not totally discard the formation of an ultramassive white dwarf. Our simulations, thus, reinforce the idea that ultramassive white dwarfs could be the end-product of the standard evolution of single heavy-weight intermediate mass stars.

Acknowledgements. Part of this work was supported by the MCYT grant AYA04094-C03-01, by the European Union FEDER funds, and by the CIRIT. We would also like to acknowledge our anonymous referee for valuable comments and criticisms. E.G. acknowledges the Japan Society for Promotion of Science for a fellowship to visit the Hokkaido University, where this project was started.

\section{References}

Abia, C., Domínguez, I., Straniero, O., et al. 2001, ApJ, 557, 126

Alpher, R. A., \& Herman, R. 1950, Rev. Mod. Phys., 22, 153

Bessel, M. S., \& Norris, J. 1984, ApJ, 285, 622

Carlberg, R. G. 1981, MNRAS, 197, 1021

Canuto, V. 1970, ApJ, 159, 641

Carr, B. J., Bond, J. R., \& Arnett, W. D. 1983, ApJ, 277, 445

Cassisi, S., \& Castellani, V. 1993, ApJS, 88, 509

Caughlan, G. R., \& Fowler, W. A. 1988, Atom. Data \& Nucl. Data Tables, 40, 283

Chieffi, A., Domínguez, I., Limongi, M., \& Straniero, O. 2001, ApJ, 554,1159

Cox, A. N., \& Stewart, J. N. 1970, ApJS, 19, 243

Christlieb, N., Bessell, M. S., Beers, T. C., et al. 2002, Nature, 419, 904
D'Antona 1982, A\&A, 115, L1

Dupuis, J., Vennes, S., \& Chayer, P. 2002, ApJ, 580, 1091

Ezer, D., \& Cameron, A. G. W. 1971, Ap\&SS, 14, 399

Finley, D. S., Koester, D., \& Basri, G. 1997, ApJ, 488, 375

Fowler, W. A., Caughlan, G. R., \& Zimmermann, B. A. 1975, ARA\&A, 13, 69

Fujimoto, M. Y., Iben, I. Jr., Chieffi, A., \& Tornambé, A. 1984, ApJ, 287, 749

García-Berro, E., Ritossa, C., \& Iben, I. 1997, ApJ, 485, 765

Gil-Pons, P., \& García-Berro, E. 2001, A\&A, 375, 87

Gil-Pons, P., \& García-Berro, E. 2002, A\&A, 396, 789

Gorieli, S., \& Siess, L. 2001, A\&A, 378, 25

Guerrero, J., García-Berro, E., \& Isern, J. 2004, A\&A, 413, 257

Heger, A., \& Woosley, S. E. 2002, ApJ, 567, 532

Heger, A., Woosley, S. E., \& Waters, R. 2000, in The First Stars, ed. A. Weiss, T. G. Abel, \& V. Hill (Berlin: Springer), 121

Heger, A., \& Woosley, S. E. 2002, in Proc. of 11th Workshop on Nucl. Astrophys., Ringberg Castle., Germany, ed. E. Müller \& W. Hillebrandt, MPA Procs., Garching, 8

Hubbard, W. B., \& Lampe, M. 1969, ApJS, 18, 297

Iben, I., Ritossa, C., \& García-Berro, E. 1997, ApJ, 489, 772

Iglesias, C. A., \& Rogers, F. J. 1993, ApJ, 412, 752

Liebert, J., Bergeron, P., \& Holberg, J. B. 2004, ApJS, in press [arXiv:astro-ph/0406657]

Limongi, M., Straniero, O., \& Chieffi, A. 2000, ApJS, 129, 625

Marigo, P. 2002, A\&A, 387, 507

Marigo, P., Girardi, C., Chiosi, C., \& Wood, R. 2001, A\&A, 371, 152

Marigo, P., Chiosi, C., \& Kudritzki, R. P. 2003, A\&A, 399, 617

Marshall, J. R., van Loon, J. T., Matsuura, M., et al. 2004, MNRAS, in press

Nakamura, F., \& Umemura, M. 2001, ApJ, 548, 19

Nieuwenhuijzen, H., \& de Jager, C. 1990, A\&A, 231, 134

Palla, F., Salpeter, E. E., \& Stahler, S. W. 1983, ApJ, 271, 632

Reimers, D. 1975, Mem. Soc. R. Sci. Liège, Ser. 6, 8, 369

Ritossa, C., García-Berro, E., \& Iben, I. 1995, ApJ, 460, 489

Ritossa, C., García-Berro, E., \& Iben, I. 1999, ApJ, 515, 381

Rogers, F. J., \& Iglesias, C. A. 1992, 401, 361

Saio, H., \& Nomoto, K. 1998, ApJ, 500, 388

Salaris, M., Domínguez, I., García-Berro, E., et al. 1998, ApJ, 486, 413

Siess, L., Livio, M., \& Lattanzio, J. 2002, ApJ, 570, 329

Tumlinson, J., \& Shull, M. 2000, ApJ, 528, L65

Weaver, T. A., \& Woosley, S. E. 1993, Phys. Rep., 227, 65

Weidemann, V. 2000, A\&A, 363, 647

Yoshii, Y., \& Saio, H. 1986, ApJ, 301, 587 